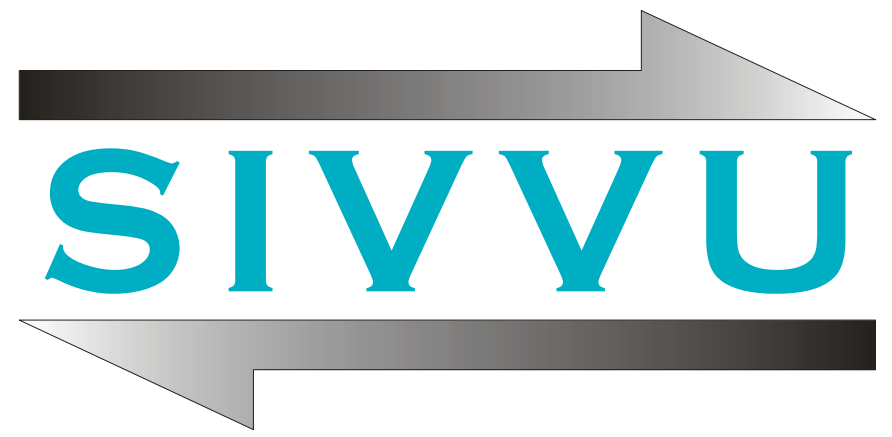

Dr. Douglas Vander Griend
Michael DeVries

Department of Chemistry \& Biochemistry

Calvin College

Grand Rapids, MI 49546-4403

Contact: sivvu@calvin.edu 

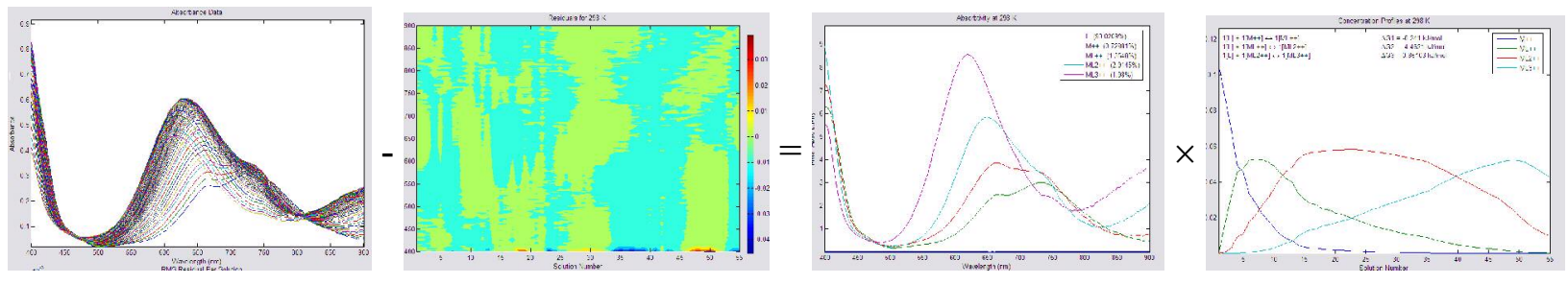

Outline

I. Getting Started
A. Overview
B. Key Features
C. Limitations
D. Loading the Program

II. The Sivvu Data Input Graphical User Interface
A. Input File
B. Chemical Species
C. Solvent
D. Activity Coefficient Model
E. Initial Spectra Guesses
F. Mass Balance and Mass Action Equations
G. Inspection Buttons: Preview, Factors \& Copy
H. Process Buttons: Save, Continue, \& Cancel

III. The Sivvu Control Graphical User Interface
A. User Control Buttons: Optimize, Calculate Error, Modify Model, Write Report, Copy Page \& Print Page
B. Plots: Absorbances, Concentration, Absorptivity, Solution Fits, Residual, \& $\Delta$ G's
C. Ignore Solutions

IV. Calculations
A. General Calculations
B. Residual Calculations

V. Appendix

A. Data for Common Solvents
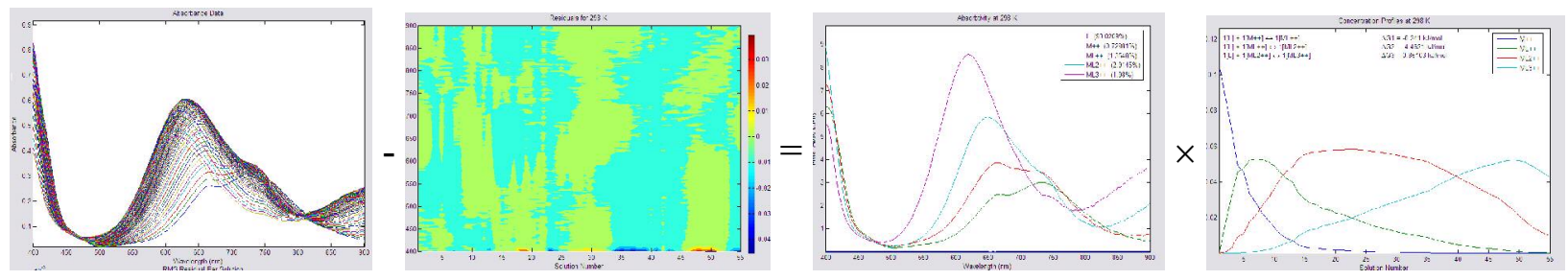


\section{Getting Started}

\section{A. Overview}

Sivvu - the name says it all (UVvis backwords). In a UVvis spectrometer, molecular species in chemical equilibrium absorb light according to Beer's Law. This program is designed to start with absorbance data for a series of solutions and ultimately extract the thermodynamic information that describes the chemical equilibria of the solutions, as well as the molar absorptivity values of all the individual species in solution.

How does it work? Specifically, the user must provide a set of chemical reactions that potentially govern all the equilibria in the system along with the raw absorbance data and information on the makeup and temperature of each solution. Sivvu then starts with initial guesses for the $\Delta \mathrm{G}_{\mathrm{rxn}}$ values, calculates the concentrations of each species in each solution and optimizes the corresponding molar absorptivity profiles for each absorbing species in the system. The thermodynamic parameters are then refined until the total residual between the observed and calculated absorbance is minimized - all in 10 to 20 seconds.

\section{B. Key Features}

- Easy to load data - one Excel based input file

- Graphical user interfaces

- Self regulation of thermodynamic model construction

- High speed analysis: full optimizations in seconds

- Concentration dependent data yields $\Delta \mathrm{G}_{\mathrm{rxn}}$ information

- Temperature dependent data yields $\Delta \mathrm{H}_{\mathrm{rxn}}$ and $\Delta \mathrm{S}_{\mathrm{rxn}}$ information

- Calculates activity coefficients according to various literature models

- Calculates standard deviation for thermodynamic parameters

- Estimates dependent and independent sensitivity of optimized $\Delta \mathrm{G}_{\mathrm{rxn}}$ values

\section{Limitations}

Sivvu is a powerful tool for studying solution phase thermodynamics and for isolating information about pure chemical compounds in a matter of minutes without the need to isolate them chemically. While a great deal of information can be extracted from a dataset about the chemistry of the solutions, the calculations are not magic. The program can only extract information as it exists in the dataset based on the mathematical relationships of chemical equilibria and Beer's Law.

Furthermore, the amount of information about a particular species is proportional to the total level of occurrence in the set of solutions. Therefore, if it only exists at a very low level in all the solutions, then the uncertainty of the molar absorptivity values for that species must be understood to be quite large.

Additionally, the resolution between two species may be poor within the dataset, particularly if their chemical or spectroscopic behaviors are comparable. For example, if both grow in with temperature, their molar absorptivity values may be irresolvable.

\section{Loading the Program}

Matlab must already be installed.

Three files are typically included with Sivvu: Sivvu.zip, SivvuManual.doc, and example.xls. Unzip the main folder into Matlab's toolbox folder, which is where all m-files for Matlab are grouped and stored. Place the example data file into Matlab's working directory, which by default is 
called 'work', but can be changed from within the Matlab environment. As a rule, the data file must be in whichever folder is designated as the working directory.

From within the Matlab environment, select 'set path' under the file menu to bring up a pathway window. If a previous version of Sivvu has been installed, remove the folder from the path directory, then add the newly unzipped folder back to the path directory. This will ensure that the all calls to m-files within Sivvu will find the proper versions.

To initialize Sivvu type 'Sivvu' into the command line of Matlab, then load in the data file by typing the name of the file into the appropriate box in the data input GUI that appears. This can also be done in one step by typing Sivvu('filename') in the command line.

\section{The Sivvu Data Input Graphical User Interface}

\section{A. The Input File(s)}

Sivvu requires exactly one input file containing all relevant experimental data. It must be the first worksheet of a Microsoft Excel spreadsheet, organized in six blocks as shown in Figure 1. The first column will contain scale and labeling information. Each subsequent column corresponds to one chemical solution. In general, the first row of each column is the temperature of the solution, followed below by the composition, then the raw absorbance data.

$\begin{array}{lrrrrr}\text { C2 } & 22 & 22 & 22 & 22 & 22 \\ \begin{array}{l}\text { M++ } \\ \text { Ligand }\end{array} & 0.1 & 0.1 & 0.1 & 0.1 & 0.1 \\ \text { BF4- } & 0 & 0.04 & 0.06 & 0.5 & 0.7 \\ 900 & 0.04369301 & 0.0470115 & 0.05090182 & 0.09089655 & 0.10069366 \\ 899 & 0.04439201 & 0.05059578 & 0.05434557 & 0.09110519 & 0.10145286 \\ 898 & 0.04186945 & 0.04922064 & 0.05312673 & 0.09252504 & 0.10289368 \\ 897 & 0.04229023 & 0.04666939 & 0.04944631 & 0.088114 & 0.10079306 \\ 896 & 0.03923234 & 0.04789623 & 0.04886965 & 0.08724912 & 0.0996298 \\ 895 & 0.04167879 & 0.04628238 & 0.04669784 & 0.08570289 & 0.09574664 \\ 894 & 0.03917151 & 0.04423087 & 0.04752459 & 0.08506723 & 0.09674081 \\ 893 & 0.03855762 & 0.04408888 & 0.04581854 & 0.08170203 & 0.09333839 \\ 892 & 0.03613525 & 0.04410593 & 0.04539375 & 0.08413263 & 0.09404626 \\ 891 & 0.03662229 & 0.04095222 & 0.04298615 & 0.08015602 & 0.09333252 \\ 890 & 0.03528416 & 0.04158612 & 0.04324281 & 0.0813244 & 0.09125246 \\ 889 & 0.03453512 & 0.03945129 & 0.04112282 & 0.07945109 & 0.08980563 \\ 888 & 0.03634429 & 0.03937295 & 0.04142636 & 0.07784642 & 0.08940628\end{array}$

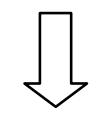

Figure 1. Archetypal Excel Spreadsheet Input File for Sivvu

Cell A1 must contain the temperature scale and the path length for the spectrometer. Simply type a K, C, or F into the cell to indicate Kelvin, Celsius or Fahrenheit, then a number (no space required) to indicate the path length in units of centimeters. If no number is provided, the assumed path length is one. The rest of the first row then, starting with cell B1 must contain the temperature data for each solution. Even if the temperature is constant, it must still be entered because it is used to calculate activity coefficients.

The next few rows contain the composition data. The first column should contain names for all of the reagents in solution, listed as ions. Figure 1 shows an example where the metal ion 
concentration is constant at $0.1 \mathrm{M}$ in each solution, while the ligand concentration varies from zero to $0.8 \mathrm{M}$. The program is designed to use units of molarity because it is directly proportional to absorbance. Other molar units of concentration can be used and the only substantial difference will be that the optimized $\Delta \mathrm{G}$ values will be slightly different, and the absorptivity values will have appropriate corresponding units.

The absorbance data is listed with the energy axis in the first column. Sivvu will annotate with wavelength in nanometers, but any scaling will work for the calculations.

Make sure that each column lines up the appropriate temperature and composition data with the corresponding absorbance data. The file should be saved into a folder which will be selected as the working directory of the Matlab interface. Any references to the solutions within Sivvu will be to the ordinal position in the data file; however, Sivvu automatically sorts the data file by ascending temperature from left to right, so solution \#1 will be the first solution at the lowest temperature. Solution \#2 will be the second solution at the lowest temperature, etc. The only limit to the number of solutions in a data file is the standard limit of 256 columns in an Excel worksheet.

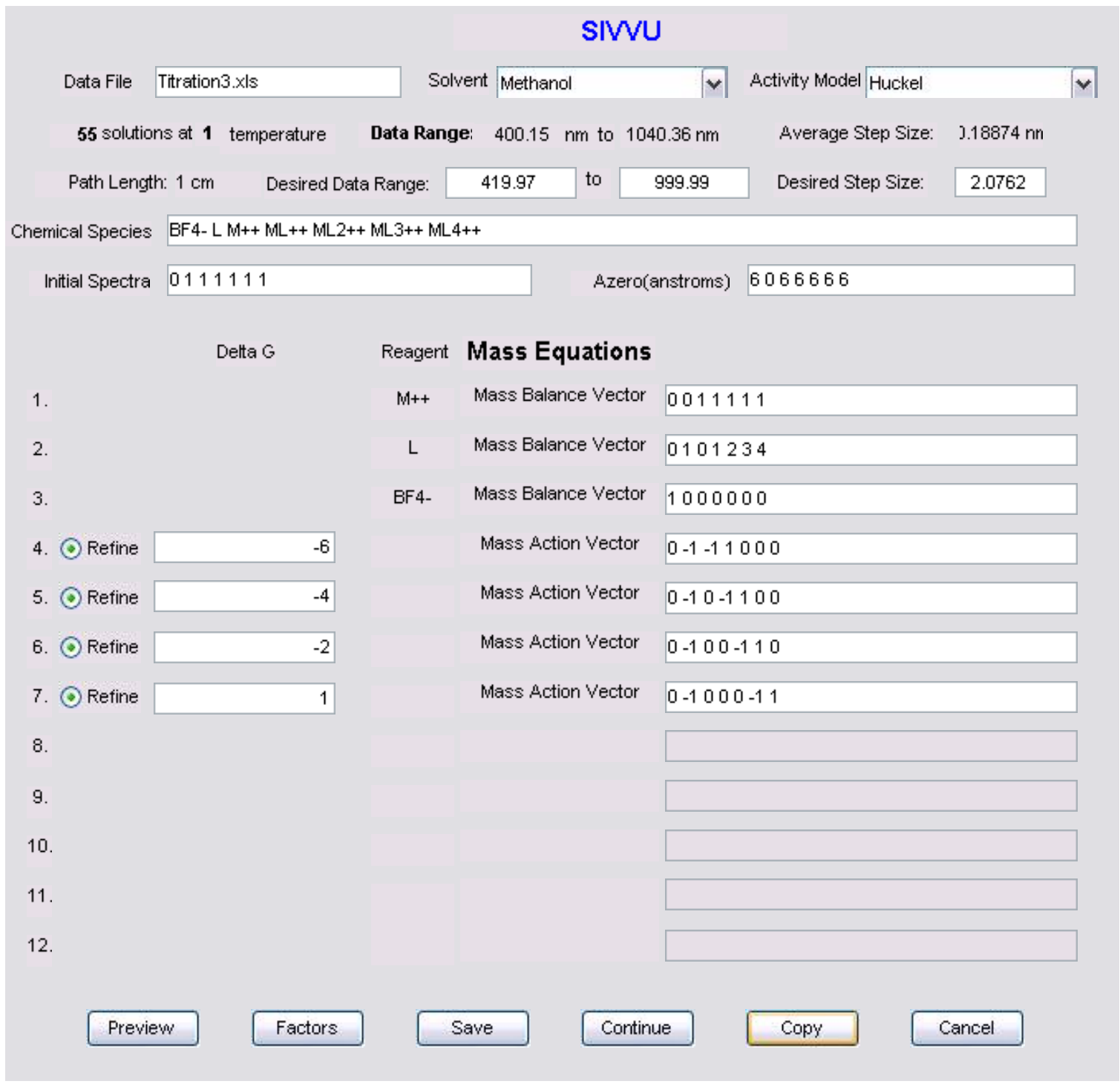

Figure 2. Sivvu Data Input GUI 
Calculations can be restricted to an arbitrary energy range and step size through the appropriate inputs on the Data Input GUI. Sivvu will restrict the data and update the inputs to the closest possible values.

If the data consists of absorbance curves at multiple temperatures, Sivvu will optimize the $\Delta \mathrm{G}_{\mathrm{rxn}}$ 's and the molar absorptivity values at each temperature independently, according to the user model.

\section{B. Chemical Species}

A list of the chemical species present in the solutions, listed as ions, is the first requirement in constructing a thermodynamic model for the data. The equilibrium concentration of each species listed here will be calculated as part of the fitting process. Therefore, in the chemical species edit box, type in a name for all species in the equilibrium reactions as well as any reagents listed in the input file that are not part of the chemical reactions. The species names are only labels and need not represent the stoichiometry of the compound. Separate each species' name with a space. Indicate the charge on each species with the correct number of plus's or minus's (e.g. Ni++). The maximum limit is 12 species.

\section{Solvent}

There is a drop-down menu available for the input of the solvent. Three possible choices already appear: Water, methanol, and $\gamma$-butyrolactone. The densities and dielectric constants for these three are already hard-coded into the program, but if another solvent is used, simply select 'Other' on the drop-down menu and a dialog box will pop up. This allows the user to enter the name, density, and dielectric constant of any solvent desired. See Appendix A for a list of useful solvent data.

\section{Activity Coefficient Model}

An activity coefficient, $\gamma$, is the proportionality constant between concentration (ideal activity) and true chemical activity, which is used in calculating equilibrium constants. There are five activity coefficient models to choose from in Sivvu, each of which uses a different equation to calculate the activity coefficients. The first model is none, which simply assigns unity to all the activity coefficients.

In the equations that follow, the variables $\mathrm{A}, \mathrm{B}, \mathrm{Z}, \mathrm{I}$, and $\mathrm{a}_{0}$ are used. $\mathrm{Z}$ is the charge vector. I is ionic strength of a solution. A and $\mathrm{B}$ are calculated at a given temperature, $\mathrm{T}$, using the solvent density, $\mathrm{d}$, and dielectric constant, $\varepsilon$, of the solvent. Their equations are:

$$
\mathrm{A}=1823928 \times \sqrt{\mathrm{d} /(\varepsilon \mathrm{T})^{3}} \quad \mathrm{~B}=\frac{50.3}{\sqrt{\varepsilon \mathrm{T}}}
$$

The equations for the other models are as follows.

$$
\begin{array}{ll}
\text { Davies: } & -\log \gamma=A Z^{2} \times \frac{\sqrt{I}}{1+\sqrt{I}}-0.3 I \\
\text { Hückel: } & -\log \gamma=\mathrm{AZ}^{2} \times \frac{\sqrt{\mathrm{I}}}{1+\mathrm{Ba}_{0} \sqrt{\mathrm{I}}}
\end{array}
$$




$$
\begin{array}{ll}
\text { Guntelberg: } & -\log \gamma=\mathrm{AZ}^{2} \times \frac{\sqrt{\mathrm{I}}}{1+\sqrt{\mathrm{I}}} \\
\text { Scatchard: } & -\log \gamma=\mathrm{AZ}^{2} \times \frac{\sqrt{\mathrm{I}}}{1+1.5 \sqrt{\mathrm{I}}}
\end{array}
$$

The $\mathrm{a}_{0}$ values, which correspond to solution radii, are taken from the ' $\mathrm{A}_{\text {zero }}$ ' edit box. The Hückel model is the only one which uses $a_{0}$ values, so if any other model is selected, the edit box for the $\mathrm{a}_{0}$ values will be disabled and filled in with a zero vector of the correct length. $\mathrm{a}_{0}$ values are only used for charged species. Some common $\mathrm{a}_{0}$ values are:

\begin{tabular}{|c|c|c|c|c|c|c|c|}
\hline Ion & $\mathrm{Co}^{2+}$ & $\mathrm{Ni}^{2+}$ & $\mathrm{Cl}^{-}$ & $\mathrm{Br}^{-}$ & $\mathrm{BF}^{-}$ & $\mathrm{NEt}_{4}^{+}$ & $\mathrm{Li}^{+}$ \\
\hline $\mathrm{a}_{0}$ & 6 & 6 & 3 & 3 & 6 & 6 & 6 \\
\hline
\end{tabular}

\section{E. Initial Spectra Guesses}

The initial spectra box takes three different types of values. Each number in this vector corresponds to a respective chemical species. If a species does not absorb, enter a zero into the box for it. Any positive number indicates that the species absorbs and that the spectrum is to be refined by Sivvu. Finally, if the species absorbs and there is a solution amidst the data that corresponds precisely to the curve of that species (at any concentration), enter the number of the solution, but as a negative value.

This last feature allows the user to enter curves of species with known molar absorptivity values. Sivvu will use them in the refinement, but not change the molar absorptivity values or the concentrations of that solution. The program assumes that any unrefined solution consists of exactly one absorbing species, and a warning message will appear if the input file is not consistent with this.

If multiple temperatures are contained within the dataset, then the initial spectra guesses must be applicable to each temperature. So, if an unrefined species exists at one temperature, it must exist as an unrefined species at all temperatures. This requires that the data file be constructed so that any unrefined species occupy the same ordinal position within each temperature set. See II.A for more details on how Sivvu rearranges the data file for multiple temperatures.

\section{F. Mass Balance and Mass Action Equations}

After the chemical species box is filled in, the appropriate number of lines for the mass balance equations and mass action equations (chemical reactions) will be enabled. The total number of mass equations needed is equal to the number of chemical species.

A mass balance equation simply defines the total amount of a reagent in the solution. One mass balance equation is required for each reagent listed in the original data file. Therefore, a mass balance vector edit box will be enabled with the name of a reagent in front of it. A vector with the same length as the chemical species list must be entered with each value indicating the number of reagent molecules in the corresponding chemical species. For example, if the list of chemical species was $\left[\mathrm{BF}_{4}-\mathrm{Cl}-\mathrm{Fe}+++\mathrm{FeCl}++\mathrm{FeCl}_{2}+\right]$, then the mass balance vector for the reagent $\mathrm{Cl}$ - would be $[0$ $\left.\begin{array}{llll}1 & 0 & 1 & 2\end{array}\right]$.

After these vectors are filled in, the mass action equations remain. This is how chemical reactions are encoded. For each, a vector with the same length as the chemical species list must be entered with each value indicating the stoichiometry of the corresponding chemical species in an arbitrary chemical reaction. A positive number in the vector indicates a product in the reaction, while 
a negative number a reactant. For example, if the species list was $\left[\mathrm{BF}_{4}-\mathrm{Li}+\mathrm{Br}-\mathrm{LiBr} \mathrm{Py} \mathrm{NiBr}_{4}-\right.$ $\left.\mathrm{NiBr}_{2} \mathrm{Py}_{2} \mathrm{NiPy}_{4}++\right]$, then the mass action vector for the creation of $\mathrm{NiBr}_{2} \mathrm{Py}_{2}$ from $\mathrm{NiBr}_{4}--$ would be

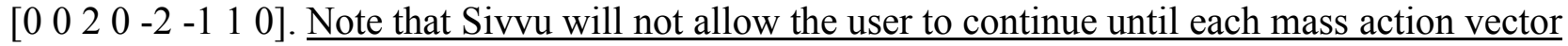
is balanced for mass and charge. Once this vector is entered, simply type an initial guess for the thermodynamic values for that reaction in the appropriate box on the left and click the radio button next to it to prepare to refine those values.

Recommendation: Even though thermodynamically it does not make a difference how the chemical species are reacted into each other, some reaction schemes may be more mathematically advantageous. Since Sivvu has no prior knowledge of the chemical species, it is advisable when entering multiple chemical reactions to make them linear in relation to each other $(\mathrm{A} \rightarrow \mathrm{B}, \mathrm{B} \rightarrow \mathrm{C}$, $\mathrm{C} \rightarrow \mathrm{D}$, etc.) rather than branching $(\mathrm{A} \rightarrow \mathrm{B}, \mathrm{A} \rightarrow \mathrm{C}, \mathrm{A} \rightarrow \mathrm{D}$, etc.). This will help eliminate any arbitrary confusion between different species.

Note: If the initial guesses at the thermodynamic values are too far off, the program may have trouble refining them because as it begins to change them, the concentrations will not shift appreciably. To check, press 'Continue' and note the concentration profiles that appear. If the graph contains only flat lines, then return to the input GUI by pressing 'Modify the Model' and change the thermodynamic parameters until the concentration profiles are no longer flat.

Note: The program has some difficulty moving initial guesses off from zero.

\section{G. Inspection Buttons: Preview, Factors \& Copy}
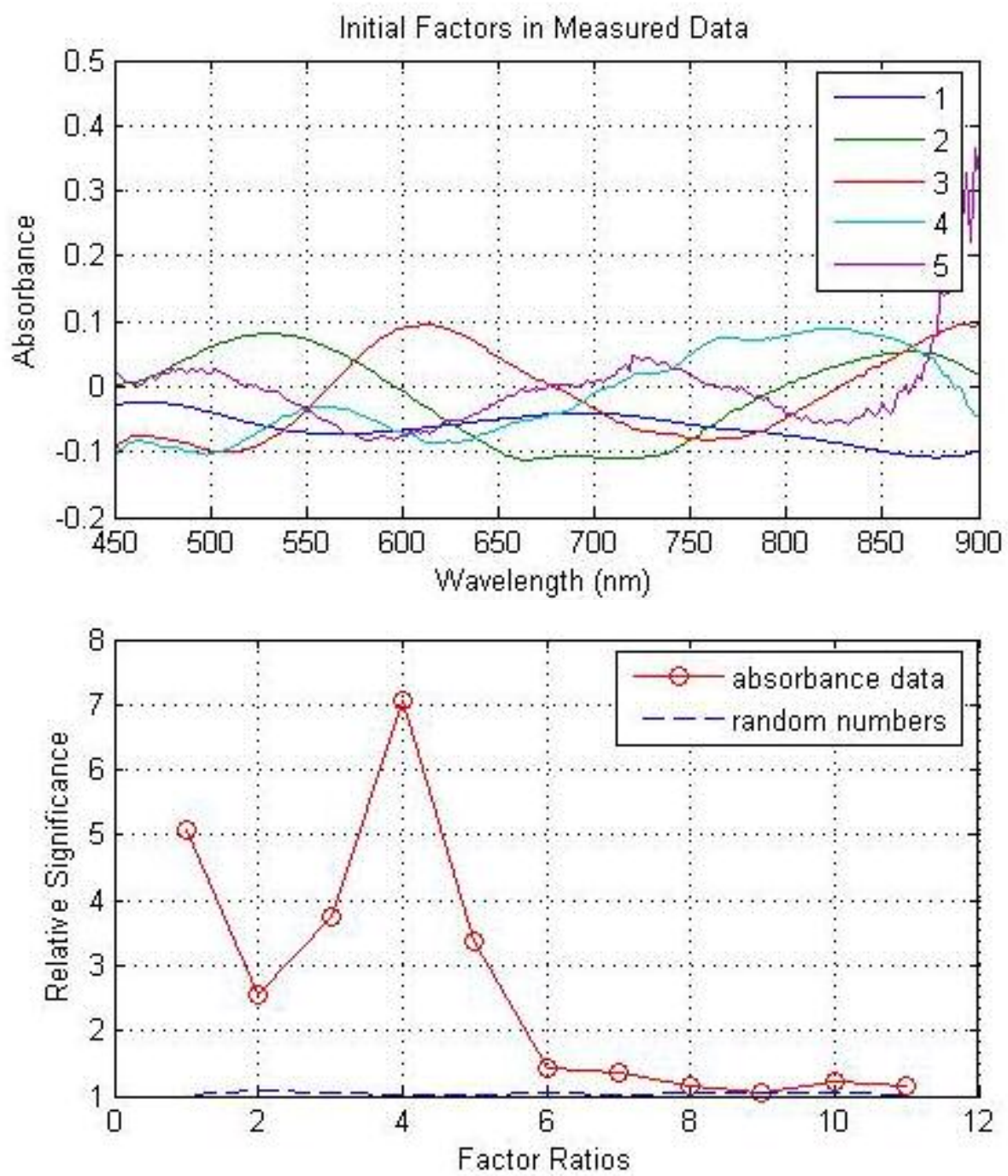

The 'Preview' button simply plots the absorbance data upon which the optimization will be based in a new window for the user to inspect and verify.

The 'Factors' button will run a protocol to assist in estimating the number of unique mathematical factors in the data which directly corresponds to the number of distinct absorbing species in the entire system. A figure with two plots will appear (see Figure 3 at left). The bottom plot shows the relative significance of the factors for the data and for an equal size data set of random numbers. The $\mathrm{n}^{\text {th }}$ point in the plot is the ratio of the $n^{\text {th }}$ largest factor to the next largest factor. The significance of the factors for a set of random numbers will be approximately one, and when

Figure 3. The additive mathematical factors in the data. 
ranked from largest to smallest, will decrease at a relatively constant rate ( $\sim 5 \%)$. Any non-random additive mathematical factors in the data will have a significance that is substantially more than that of the next most significant factor. Therefore, the plot of relative significance will drop down to the random baseline after all the true factors have been accounted for. The data used in Figure 3 clearly shows five significant factors because the fifth factor is over three times larger yet than the sixth, but after that all the factors are about the same size and close to unity.

The top plot shows the first few additive mathematical factors in the data. You may recognize some spectroscopic features, but because these curves are not restricted in any way, they will include negative numbers and other mathematical artifacts.

\section{H. Process Buttons: Save, Continue, Copy, \& Cancel}

The 'Save' button will first check over all of the vectors to make sure they are all of the same length, and notify the user if any of the chemical reactions are not balanced. It will then pull in all of the information entered, place it in a Matlab variable structure called Sivvu_input, and create a file with this information in it, which is saved in the current directory. The file created has the extension '.mat' and its name is the same as that of the data file. Once this file is saved, Sivvu will load it every time it processes the data file of the same name. To modify it, simply load it into Sivvu, make the necessary changes and save the file again. The mat file will be overwritten.

The 'Continue' button initiates several calculations on the data. The concentration profiles of all the species listed are calculated from the initial thermodynamic data provided $\left(\Delta \mathrm{G}_{\mathrm{rxn}}\right.$ 's). Then the spectra of the absorbing species to be refined are optimized with a least squares fitting protocol. Finally, the Sivvu Data Input GUI will be replaced with the Sivvu Control GUI. The 'Continue' button protocol will also automatically save the user input in the same manner as the 'Save' button if and only if the mat file does not yet exist.

The 'Copy' button places a figure file of the Sivvu window on the computer clipboard from where it can be pasted into other programs.

The 'Cancel' button closes Sivvu and clears all variable information from the Matlab environment. Any unsaved information will be lost.

\section{The Sivvu Control Graphical User Interface}

Figure 4 shows four views of the Control GUI, which contains a set of user controls on the left, two sets of axes, and some key information in the bottom left. The concentration profiles will be immediately displayed on the main axes, and the bottom set of axes is immediately employed to show a bar graph of the root-mean-square residual for individual solutions in the system as defined with the initial guesses. At this point several options are available.

\section{A. User Control Buttons: Optimize, Calculate Error, Modify Model, Write Report, Copy Page \& Print Page}

The 'Optimize' button in the upper left corner of the screen begins the main function of the program; it will optimize the thermodynamic values $\left(\Delta \mathrm{G}_{\mathrm{rxn}}\right.$ 's $)$ starting with the initial guesses and narrow in on the values that will produce the least residual in the system. Specifically, the rootmean-square of the residuals (the difference between the observed and calculated values) is minimized. The values will then be placed in a Matlab structure called Sivvu_result.RefinedGvalues. This process will normally take less than a minute as Sivvu can typically check 10 sets of thermodynamic parameters per second. The only output during the optimization will be visible in the Matlab command window. When complete the absorptivity values will automatically be plotted on 
the main graph. To end the optimization procedure prematurely, go to the command window and simultaneously press 'Ctrl' and ' $\mathrm{C}$ '. This will terminate all procedures running in Matlab.

The sliders at the top left of the interface allow the user to adjust the tolerances on the search for the minimum residual. Sivvu will optimize the $\Delta \mathrm{G}_{\mathrm{rxn}}$ 's until the optimal values are constant within the specified tolerance and the root-mean-square residual is minimized within its tolerance.

If you want the calculations to go even faster, select the suppress output check box in the upper left corner. No running output will be written to the command line and so the calculation goes about twice as fast.

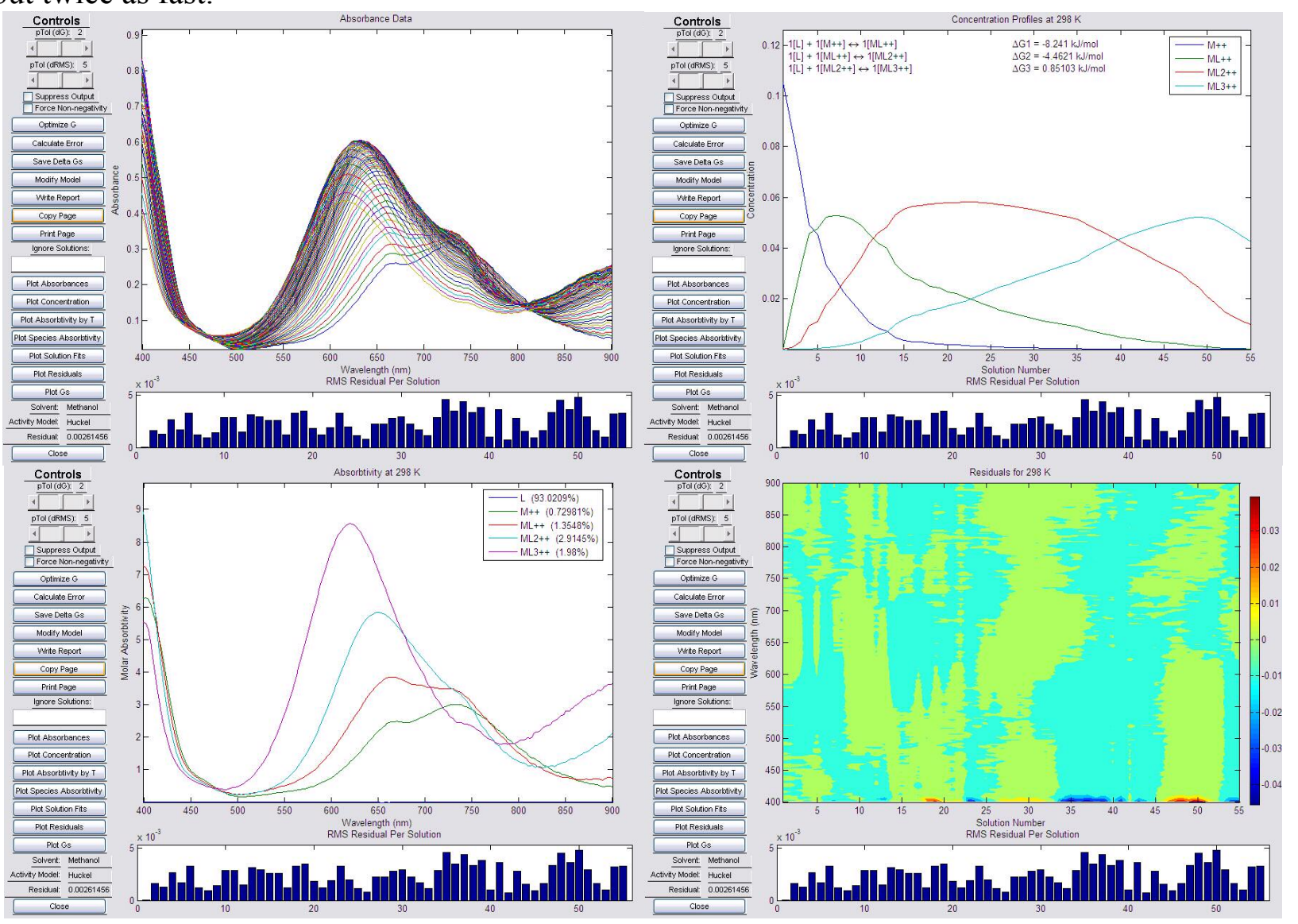

Figure 4: Four examples of the plot window in the Sivvu Control GUI

The 'Calculate Error' button launches an extensive protocol to calculate the standard deviation in the optimized free energies in three different ways. First, $\Delta \mathrm{G}_{\mathrm{rxn}}$ values are re-optimized repeatedly (20 times) after $10 \%$ of the wavelengths are ignored. Then the standard deviation of the re-optimized values for each free energy value is calculated. The running output in the command line is suppressed during these calculations to save time.

Second, independent error is calculated by sequentially changing each $\Delta \mathrm{G}_{\mathrm{rxn}}$ to a value one $\mathrm{kJ} / \mathrm{mol}$ greater and one less than the optimized value, and then calculating the increase in the RMS residual. This is a measure of how much each $\Delta \mathrm{G}_{\mathrm{rxn}}$ independently affects the quality of fit.

Finally, the dependent error is calculated in the same way as the independent error, except that the unchanged $\Delta \mathrm{G}_{\mathrm{rxn}}$ values are re-optimized before the increase in RMS residual is calculated. This then measures how much a $\Delta \mathrm{G}_{\mathrm{rxn}}$ in conjunction with the other values affects the quality of fit. 
The results of all error calculations are written to the command line and stored in Sivvu_result. They are also included when a report is generated.

The new refined thermodynamic values can then be saved into the mat file of the corresponding data set by pressing the 'Save Delta Gs' button. The next time the data file is loaded into the Sivvu Data Input GUI, the refined values will be in place of the original initial guesses, so the program will start any new refinement with more accurate values.

At any time the 'Modify Model' button will send the user back to the Sivvu Data Input GUI. Initially, all of the data stored in the mat file for the system will be loaded up, and any necessary changes can be made, or a new experiment can be started.

Note: Pressing the 'Modify Model' button will clear the Sivvu_result variable structure and thus lose any data generated by optimization.

The 'Write Report' button will create an excel spreadsheet containing relevant information about the thermodynamic model, the thermodynamic values and their standard deviations (if error calculations have been done), and the molar extinction coefficients for the absorbing species. The creation of this file will take approximately ten seconds, and it will be located in the working directory. The name will be the original data file name with the suffix 'report'.

The 'Copy Page' button will copy a screen shot of the Control GUI onto the windows clipboard that can be pasted into other windows programs. The 'Print Page' button will bring up the printer dialog box for printing out a hard copy of the GUI screen shot.

\section{B. Plots: Absorbances, Concentration, Absorptivity, Solution Fits, Residual, \& $\Delta$ G's}

There are many options for plots to show on the large axes (see Figure 3 for examples). The first is to 'Plot Absorbances'. This will simply take the portion of the original data matrix that is used for the refinement and plot the absorbance data found there. The second option is to 'Plot Concentrations', which will plot the calculated equilibrium concentration profiles (concentration as a function of solution) for each of the absorbing species in the system. The 'Plot Absorptivity' buttons will plot the molar absorptivity values in one of two possible ways: either by temperature or by absorbing species. The 'Plot Solution Fits' button will display the observed and calculated data for individual data curves. Repeated calls with this button will cycle through all of the curves in the dataset. The 'Plot Residuals' button will calculate all the residuals in the system and display them as a color contour map. Finally, the 'Plot Gs' button will generate a plot of the $\Delta \mathrm{G}$ values for a reaction versus temperature. If the data consists of multiple temperatures the line of best fit will also be plotted. The slope and intercept of this line are $-\Delta \mathrm{S}$ and $\Delta \mathrm{H}$ respectively, and are displayed on the graph. Successive calls with this button will cycle through each reaction in the user model.

For data at multiple temperatures, successive calls to any of these buttons display the data from the next temperature dataset.

\section{Ignore Solutions}

Next, if the bar graph of residual per solution at the bottom reveals that one or more solutions have an unusually high amount of residual relative to the others, Sivvu provides the option to ignore these solutions in its calculations. Type in the number or numbers of the solutions to be ignored in the ignore solutions edit box and press enter. From that point on, Sivvu will leave these solutions out of all calculations. Therefore optimization of thermodynamic values will occur with these solutions ignored. To reinstate the solution(s), simply clear out the ignore solutions edit box and press enter, and Sivvu will be ready to optimize with that data included. 


\section{Calculations}

\section{E. General Calculations}

Sivvu makes use of two major mathematical relationships in chemistry. The first is the BeerLambert law for spectroscopic data. Because it is an additive law it can be expanded to include multiple species. It can be conveniently written in matrix form according to the following equation.

$$
\operatorname{Abs}_{\mathrm{p}}^{\mathrm{n}}=\sum_{\mathrm{m}} \varepsilon_{\mathrm{m}}^{\mathrm{n}} \mathrm{C}_{\mathrm{p}}^{\mathrm{m}}
$$
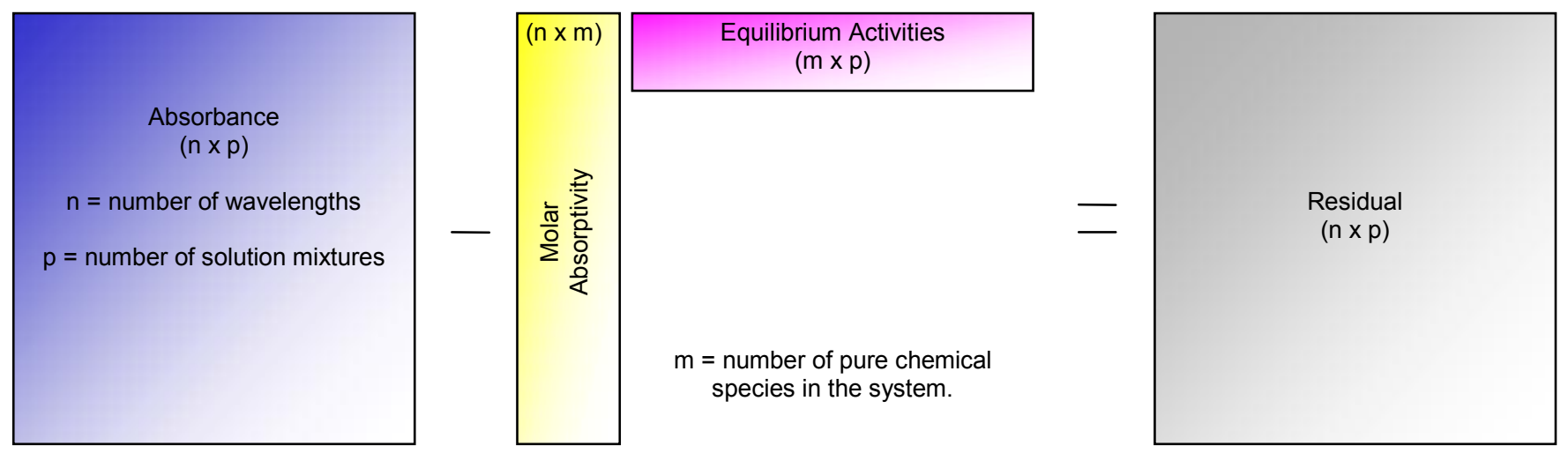

Figure 5: The Beer-Lambert Law in schematic matrix form.

The second mathematical relationship is that of chemical potential which leads to equilibrium concentrations. It simply describes the chemical potential of the system, which is ultimately minimized at equilibrium, in terms of activity, which itself can be deduced in a variety of ways. The most straightforward leads directly to the familiar equilibrium expression of 'products over reactants':

$$
\mu_{\mathrm{i}}=\mu_{\mathrm{i}}^{\circ}+\mathrm{RT} \ln \mathrm{A}_{\mathrm{i}}
$$

Here $\mu_{\mathrm{i}}{ }^{\circ}$ is calculated from the $\Delta \mathrm{G}_{\mathrm{rxn}}$ values and the activity, $\mathrm{A}$, is calculated from the concentration term in a variety of ways (see II.D).

The program begins by using the initial $\Delta \mathrm{G}_{\mathrm{rxn}}$ values for the equilibrium reactions to calculate equilibrium activities and concentrations that satisfy the mass and charge balance parameters. With matrices of absorbance data and concentrations, Sivvu can then directly optimize the molar absorptivity of each absorbing species at each wavelength by least squares minimization. This calculation amounts to solving $(\mathrm{S} \times \mathrm{N})$ simultaneous linear equations for $(\mathrm{M} \times \mathrm{N})$ molar absorptivity values, where $\mathrm{S}$ is the number of solutions, $\mathrm{M}$ is the number of species, and $\mathrm{N}$ is the number of wavelengths.

Now, having found spectral and concentration data for each species, Sivvu calculates the difference between the measured absorbance data and the product of the optimized molar absorptivity values with the calculated concentrations. The root-mean-square is taken of these residuals to ascertain the quality of fit for the data. Finally, Matlab searches for thermodynamic values that minimize the total root-mean-square residual. 
Within the Matlab environment, Sivvu works with three variable structures for storing all of the important values used during the calculations. Sivvu_data contains all of the important information from the input data file, and once populated this structure is not changed unless a new data file is called. Sivvu_input contains user-defined information on the thermodynamic model, and Sivvu result contains the data actually used in calculations, trimmed down according to user specifications and separated into distinct data sets for each temperature, as well as the output from the optimization calculations.

There are also several other variables that exist while Sivvu is being used. They include filename, which contains the name of the data file, initconc, which is the initial guess at the equilibrium concentrations, and track, which records which buttons have been pressed in the User Control GUI.

\section{F. Residual Calculations}

The primary error calculation is based on the residuals - the point by point difference between the calculated and measured absorbance values. The squares of these values are averaged. The square root of this average is what is presented as total RMS error, and this is the value that is minimized. The program will also print out several other numbers which quantify the degree of fit. The final lack of fit is the variance, defined as RMS error divided by the RMS absorbance. The final

$\mathrm{R}^{2}$ is defined as one minus the square of the variance, and the standard deviation in absorbance values is the standard deviation of the residuals.

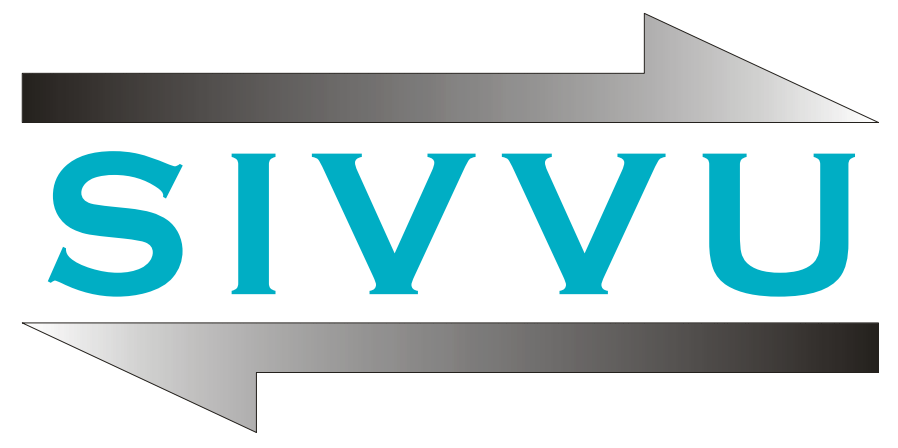




\begin{tabular}{|c|c|c|c|c|c|}
\hline \multicolumn{6}{|c|}{ Appendix A: Data for Common Solvents } \\
\hline Name & Structure & $\begin{array}{c}\text { Density } \\
(\mathrm{g} / \mathrm{ml})\end{array}$ & $\begin{array}{l}\mathrm{BP} \\
\left({ }^{0} \mathrm{C}\right)\end{array}$ & $\begin{array}{c}\text { dipole } \\
\text { moment }\end{array}$ & $\begin{array}{l}\text { dielectric } \\
\text { constant }\end{array}$ \\
\hline water & $\mathrm{H}-\mathrm{OH}$ & .998 & 100 & 1.85 & 80 \\
\hline 1-butanol & $\begin{array}{c}\mathrm{CH}_{3} \mathrm{CH}_{2} \mathrm{CH}_{2} \mathrm{CH}_{2-} \\
\mathrm{OH}\end{array}$ & .81 & 118 & 1.66 & 17.8 \\
\hline 1-propanol & $\mathrm{CH}_{3} \mathrm{CH}_{2} \mathrm{CH}_{2}-\mathrm{OH}$ & .803 & 97 & 1.68 & 20.1 \\
\hline acetic acid & $\mathrm{H}_{3} \mathrm{C}-$ & 1.049 & 118 & 1.74 & 6.15 \\
\hline acetone & & .786 & 56 & 2.88 & 20.7 \\
\hline acetonitrile & $\mathrm{H}_{3} \mathrm{C}-\mathrm{C} \equiv \mathrm{N}$ & .786 & 81 & 3.92 & 36.6 \\
\hline benzene & & .879 & 80 & 0 & 2.28 \\
\hline$\gamma$-butyrolactone & & 1.12 & 204 & 4.4 & 39.1 \\
\hline carbon tetrachloride & $\mathrm{CCl}_{4}$ & 1.594 & 76 & 0 & 2.24 \\
\hline $\begin{array}{l}\text { diemthyl sulfoxide } \\
\text { (DMSO) }\end{array}$ & & 1.092 & 189 & 3.96 & 47.2 \\
\hline diethyl ether & $\mathrm{CH}_{3} \mathrm{CH}_{2} \mathrm{OCH}_{2} \mathrm{CH}_{3}$ & .713 & 35 & 1.15 & 4.34 \\
\hline ethanol & $\mathrm{CH}_{3} \mathrm{CH}_{2}-\mathrm{OH}$ & .789 & 78 & 1.69 & 24.3 \\
\hline ethyl acetate & $\stackrel{\stackrel{O}{\|}}{\mathrm{H}_{3} \mathrm{C}-\mathrm{C}-\mathrm{OCH}_{2} \mathrm{CH}_{3}}$ & .894 & 78 & 1.78 & 6.02 \\
\hline formamide & & 1.13 & 210 & 3.73 & 109 \\
\hline formic acid & $\mathrm{H}-\mathrm{C}-\mathrm{OH}$ & 1.22 & 100 & 1.41 & 58 \\
\hline hexane & $\mathrm{CH}_{3}\left(\mathrm{CH}_{2}\right)_{4} \mathrm{CH}_{3}$ & .655 & 69 & 0 & 2.02 \\
\hline methanol & $\mathrm{CH}_{3}-\mathrm{OH}$ & .791 & 68 & 1.70 & 33 \\
\hline methyl ethyl ketone & $\mathrm{H}_{3} \mathrm{C}-\mathrm{C}-\mathrm{CH}_{2} \mathrm{CH}_{3}$ & .805 & 80 & 2.78 & 18.5 \\
\hline methylene chloride & $\mathrm{CH}_{2} \mathrm{Cl}_{2}$ & 1.326 & 40 & 1.60 & 9.08 \\
\hline $\begin{array}{l}\text { N,N-dimethylformamide } \\
\text { (DMF) }\end{array}$ & $\mathrm{H}^{\prime 4} \mathrm{H}$ & .944 & 153 & 3.82 & 38.3 \\
\hline $\begin{array}{l}\text { Tetrahydrofuran } \\
\text { (THF) }\end{array}$ & & .886 & 66 & 1.63 & 7.52 \\
\hline
\end{tabular}

